
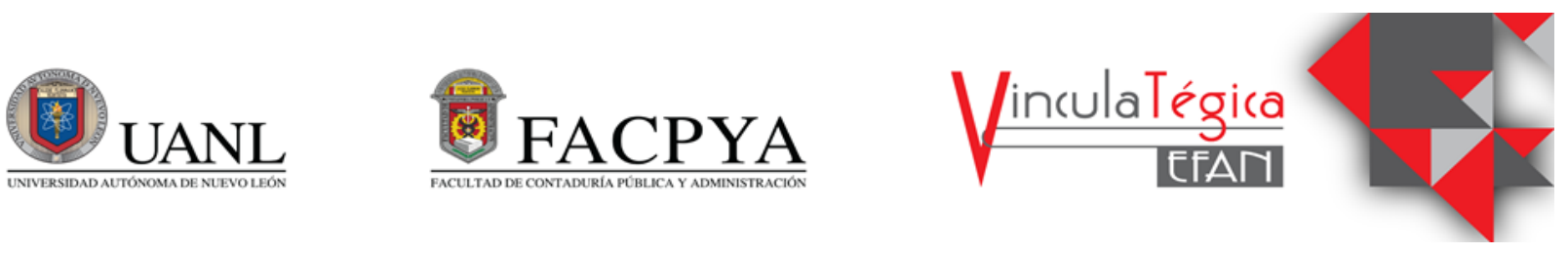

\title{
El síndrome Boreout y su incidencia en la gestión de capital humano
}

\author{
Blanca Nelly Rodríguez Garza ${ }^{1}$ e Ivon Abigail González Garza ${ }^{2}$ \\ ${ }^{1}$ Universidad Autónoma de Nuevo León, Facultad de Contaduría Pública y Administración Monterrey, Nuevo \\ León, México, bngarza6@gmail.com, Av. Universidad S/N, Col. Ciudad Universitaria, (+52) 8183204080 \\ ${ }^{2}$ Universidad Autónoma de Nuevo León, Facultad de Contaduría Pública y Administración Monterrey, Nuevo \\ León, México, ivonne.gzz.gza@gmail.com, Av. Universidad S/N, Col. Ciudad Universitaria, (+52) 818320 \\ 4080
}

4080

\begin{abstract}
Información del artículo revisado por pares
Fecha de aceptación: junio-2021

Fecha de publicación en línea: diciembre-2021

DOI: https://doi.org/10.29105/vtga7.1-169
\end{abstract}

\begin{abstract}
Resumen
Abstract

Durante la presente investigación se pretende abordar desde una perspectiva teórica un fenómeno laboral que repercute negativamente en el rendimiento de muchos trabajadores, el "Síndrome Boreout". La obligación de estar en el trabajo durante horas sin saber qué hacer es una situación desalentadora para cualquier empleado. El boreout es lo contrario del burnout (Síndrome del quemado) y se compone de: infraexigencia, aburrimiento y desinterés.

El Síndrome Boreout puede ocasionar lo que los expertos en recursos humanos han llamado "presentismo laboral", acudir al puesto de trabajo, pero dedicarse a realizar otras tareas personales, que no son propias de la función de su empleo. Uno de los mayores problemas en el área de gestión de personal es el relacionado a síntomas que manifiesta el trabajador, y que puede desencadenar en una enfermedad. Ante la poca investigación científica que hasta el momento se ha realizado, surge la siguiente interrogante: ¿cuáles son los enfoques teóricos, tendencias y perspectivas, del síndrome laboral Boreout así como su incidencia en la gestión de capital humano? Después de una exhaustiva aproximación teórica, se concluye que el Boreout, es un riesgo psicosocial en las organizaciones que afecta al personal desmotivado que realiza tareas repetitivas en su trabajo o no experimenta progreso en el mismo, se confirma como un estado

En In this research, we will analyze from a theoretical perspective a phenomenon found in a working environment which affects the performance of many people, this phenomena is called "Boreout Syndrome". The duty of spending hours at work without knowing what to do is a discouraging situation for any worker. In the opposite, the "Burnout syndrome" is composed by the feeling of being able to perform more than required, boredom, and lack of interest.

The Boreout Syndrome may cause what the experts have called "presenteeism in the workplace", that is when people are physically at work but they are not actually working, they do other personal activities without being productive. One of the biggest problems in the area of personnel management is the relation to symptoms that the worker manifests, and which may lead to illness. In face of the little scientific investigation that has been done so far, the follow question arises: which are the theoretical approaches, tendencies and perspectives, of the laboral Boreout syndrome as well as its incidence in the human capital management? After an exhaustive theoretical approximation/approach, it is concluded that the Boreout, is a psychosocial risk
\end{abstract}


dañino a nivel personal y organizacional. Se finaliza con propuestas de prevención, pretendiendo que este estudio constituya la base para una futura investigación cuantitativa. in the organization that affects unmotivated personnel who perform repetitive tasks in their work or do not experiment progress in it, it is configured as a harmful situation on a personal and organizational level. It ends with prevention proposals, pretending that this study constitutes the basis for a future quantitative investigation.

Keywords: Boreout syndrome, Infra-exigency / infra-demand, Boredom, Disinterest.

jornadas y tiempos laborales, y los lugares para desempeñar su trabajo, en consecuencia, los empleos empezaron a responder a necesidades distintas. Estas condiciones nuevas suscitaron la aparición de profesionales que se especializaran en entender y predecir el comportamiento de los individuos dentro del ámbito laboral, así como en el análisis de las condiciones en que este se desarrolla. Esta comprensión favoreció el surgimiento de novedosas formas de mediar tanto en el empleado como en el ámbito laboral, para preservar la productividad y favorecer el bienestar de los trabajadores, comprendiendo la prevalencia de bienestar y eficiencia en términos de reciprocidad (Robbins y Coulter, 2018).

Una vez que se identificó la necesidad del capital humano dentro de las compañías, y sabiendo que era imposible explotar su gente sin que esta renunciara o se viera afectada por largas jornadas de trabajo que dejaban exhausta su fuerza laboral, fue necesario implementar un nuevo departamento que pudiera ver por las necesidades de los empleados, así como asegurarse de la contratación del talento requerido por la empresa, este departamento es el de Recursos Humanos, que de acuerdo con Robbins y Coulter, (2018), es el encargado de garantizar a la empresa la correcta selección del personal que se va a destinar para cada vacante que llegue a abrirse dentro de la organización. Así 
mismo se encarga de desarrollar un plan específico de gestión de capital humano con el que se puedan identificar perfiles adecuados para cada puesto, o, en caso de ser necesario, el diseño de nuevas posiciones con las que se pueda llevar a cabo un mejor desempeño por parte de los departamentos administrativos o de producción.

Ante tales circunstancias históricas, se presentan una serie de condiciones que evidencian la manifestación de diversas patologías en el espacio laboral y problemas particulares ocasionados por la especialización del trabajo (Bahamondes, 2018). En respuesta a tales hechos históricos nace la Psicología Industrial, que desde ese momento se ocupa del nombrado riesgo psicosocial en el trabajo, conceptualizado como todas aquellas condiciones que tienen que ver con la organización laboral, las condiciones del ámbito de trabajo, el contenido del puesto y el desempeño de las actividades y funciones propias, y que tienen el efecto de afectar tanto el desarrollo del empleo, como la salud y el bienestar de las personas que lo realizan (Peiró, 2008).

$\mathrm{Al}$ analizar los riesgos psicosociales en el trabajo, a nivel internacional se han detectado algunos síndromes vinculados a una función laboral, por ejemplo, el Síndrome de Burnout, uno de los más frecuentes en las organizaciones. Es conocido como el fenómeno de "quemarse en el trabajo" y deriva en estrés y es comúnmente derivado de una sobrecarga laboral. La mayoría de las investigaciones se centran en el síndrome de Burnout, aunque últimamente se ha reconocido otra patología que ha aparecido en respuesta a una mínima exigencia en el empleo, y se ha denominado el Síndrome de Boreout (Rothlin y Werder, 2011).
El síndrome de aburrimiento laboral disminuye la productividad de las organizaciones, por lo que resulta en una serie de costos económicos. De hecho, en algunas empresas estadounidenses se ha reportado que los costos monetarios debidos a los bajos niveles de productividad asociados al síndrome fueron mayores a los 750 millones de dólares al año, lo que fue equivalente a más de 5 mil dólares por trabajador al año (Cabrera Noriega, 2014). Debido a los altos costos que representa el síndrome de aburrimiento laboral para las organizaciones, se han comenzado a investigar los factores de riesgo asociados al síndrome. No obstante, la ausencia de instrumentos de medición ha dificultado su evaluación, por lo que la literatura a nivel nacional e internacional es escasa.

En un estudio empírico realizado por los investigadores García, Villavicencio y García, de la UNAM, en el 2019, con el propósito de analizar los grados del síndrome de aburrimiento laboral entre trabajadores de México pertenecientes a las generaciones baby boomer, $\mathrm{x} y$ millennial, se descubrió que los niveles del síndrome de Boreout fueron primordialmente bajos $y$ no se diferenciaron entre generaciones. Los autores del estudio admiten un posible sesgo en los resultados y reconocen la necesidad de continuar implementando métodos para valorar el síndrome de boreout con la objetivo de diseñar intervenciones efectivas para su tratamiento $\mathrm{y}$ medidas preventivas para el mismo.

En el presente estudio se pretendió hacer un análisis de la conceptualización teórica, las orientaciones y criterios acerca del síndrome laboral Boreout, así como su incidencia en la gestión de recursos humanos. 


\section{MARCO TEÓRICO}

\subsection{Estudios previos relacionados con} el síndrome del Boreout

En una investigación llevada a cabo por Dan Malachowski (Salary.Com., 2005) sobre "El tiempo perdido en el trabajo cuesta miles de millones a las empresas"; se aplicó una encuesta, sobre el tema del despilfarro del tiempo en el trabajo. De este sondeo se arrojaron datos sumamente interesantes, pues el 33,2 \% de los trabajadores, indicó que no se encontraba lo suficientemente ocupado en su empleo y que se sentía poco exigido en el mismo. Esta indagación, muestra que los trabajadores infra exigidos dedican diariamente dos horas, en sus puestos de trabajo, para realizar tareas privadas.

De acuerdo con Malachowski, la lista de las 10 actividades en las que más se desperdicia el tiempo en las compañías de Estados Unidos se aprecia en la siguiente Tabla:

Tabla 1

Top de Diez Actividades para el desperdicio del tiempo

\begin{tabular}{|l|l|}
\hline Navegar en ciberespacio (uso personal) & $44,70 \%$ \\
\hline Socializar con otros empleados en el lugar de trabajo & $23,40 \%$ \\
\hline Realización de negocios personales & $6,80 \%$ \\
\hline Entretenimiento & $3,90 \%$ \\
\hline Realizar mandados fuera de lugar de trabajo & $3,10 \%$ \\
\hline Hacer llamadas telefónicas personales & $2,30 \%$ \\
\hline Aplicar para otros empleos & $1,30 \%$ \\
\hline Planear eventos personales & $1 \%$ \\
\hline Llegar tarde/Irse tarde & $1 \%$ \\
\hline Otras & $12,50 \%$ \\
\hline
\end{tabular}

Fuente: Estudio de Dan Malachowski para galaxi.com y AOL “Wested Time at Work Costing Companies Billions”, Julio de 2005 , publicado en Salary.com.

Además, dicho estudio ofrece un listado de las observar a continuación: cinco principales excusas para perder el tiempo en el trabajo, las cuales se pueden

Tabla 2

Top Cinco de Excusas para el desperdicio del tiempo

\begin{tabular}{|l|l|}
\hline No tener suficiente trabajo para hacer & $33,20 \%$ \\
\hline Paga menor a la cantidad de trabajo asignado & $23,40 \%$ \\
\hline Distracción por compañeros de trabajo & $14,70 \%$ \\
\hline Falta de tiempo después del trabajo & $12 \%$ \\
\hline Otros & $16,70 \%$ \\
\hline
\end{tabular}

Fuente: Estudio de Dan Malachowski para galaxi.com y AOL "Wested Time at Work Costing Companies Billions", Julio de 2005, publicado en Salary.com.

Los suizos Philippe Rothlin y Peter R. Werder, en el 2007, empezaron a interesarse en el estudio de esta problemática, analizando la incidencia de malgastar tiempo en el trabajo, partiendo de diversas investigaciones, principalmente con fundamento en la encuesta aplicada más de cien mil trabajadores, por Dan Malachowski en el 2005, concluyendo que el
$33 \%$ de los empleados encuestados indicaba que su empleo no le representaba exigencia alguna y que aproximadamente transcurrían dos horas al día desperdiciando el tiempo ( $\sin$ considerar las horas permitidas dentro de la jornada para consumo de alimentos y para descansar). Ese mismo estudio constató que al $15 \%$ de los trabajadores en oficinas a nivel 
mundial, le aburre su empleo. A través de otro instrumento, esta vez de la consultora TMI (Transformación Manejada con Inspiración), se investigó que el $80 \%$ de los empleados no se sentía comprometido con su trabajo, por lo que se mantenía sin interés aparente al éxito y/o fracaso de la empresa para la que trabajaba (Rothlin y Werder, 2011).

En el mismo tenor, la Gallup Organization encontró que el $87 \%$ de los empleados de nacionalidad alemana experimentaban poco o nulo vínculo a su organización. Algunos estudiosos en sus trabajos de investigación sobre este fenómeno estiman que una de las razones, consiste en que siete de cada diez encuestados no ocupan un cargo con el que se sientan identificados o que realmente les guste (Rothlin y Werder, 2011). Bajo el mismo supuesto de que en promedio una persona vive gran parte de su tiempo en el trabajo, y que lo que este le brinda se puede transformar en factores que reditúen en calidad de vida y bienestar, o bien, coadyuvar al deterioro de estos, repercutiendo de forma directa en la salud laboral, Rothlin y Peter Werder en 2007, empiezan a utilizar el término Síndrome de Boreout, haciendo referencia al aburrimiento crónico. Dichos autores definen este fenómeno laboral como la obligación de estar en el lugar de trabajo durante horas sin saber qué hacer; lo cual para un trabajador resulta desalentador y desmoralizador, por lo que su rendimiento y la predisposición del recurso humano afectado por este síndrome, se ven afectadas negativamente.

La exploración de los estudios llevados a cabo hasta el momento proporciona pruebas de la manifestación de factores de riesgo psicosocial en el entorno laboral que se pueden determinar como factores de aparición y/o desarrollo del síndrome de Boreout. Las investigaciones hacen posible considerar las consecuencias que tiene dicho fenómeno tanto para las empresas como para los trabajadores afectados, por lo que se justifica como una razón suficiente para preguntarse si en el ambiente laboral se cuenta con suficiente información relacionada con el fenómeno, su desarrollo, y las circunstancias que lo provocan y lo sostienen en el tiempo.

Ante la relevancia del síndrome y sus consecuencias para una acertada gestión y desenvolvimiento del Capital Humano en las organizaciones, se evidencia una muy escasa documentación científica sobre este fenómeno laboral repercute en un mayor número de empleados y que desencadena en una depresión y falta de autoestima. Philippe Rothlin y Peter Werder (2011) son los primeros en describir este síndrome utilizando el término Boreout para identificarlo a partir de 2007. Estos autores señalan que con frecuencia esta problemática se presenta cuando un gerente no delega, ya sea por la gratificación que significa llevar a cabo más funciones, o bien porque se interesa más en desempeñar cualquier tipo de actividades excepto aquellas que son monótonas, repetitivas e irrelevantes. Los propósitos al intentar definir el síndrome Boreout, propuestas por Rothlin y Werder, continúan siendo el cimiento sobre el que se han efectuado subsecuentes estudios, pero no se ha presentado una argumentación diferente en términos de conceptualización, de tal fenómeno. La interrogante es entonces ¿cuáles son los enfoques teóricos, perspectivas y tendencias del síndrome laboral del aburrimiento crónico (Boreout)?

De acuerdo con Pedro Gil-Monte, (2014), es evidente el acicalamiento negativo que significa para una organización el hecho de que sus trabajadores experimenten tedio crónico en su empleo; el capital humano se vería desperdiciado, los empleados se transforman en talentos infrautilizados y la organización perdería eficiencia y competitividad. Además de las consecuencias negativas a nivel organizacional, el Síndrome de Boreout debe ser entendido como un estado que puede provocar en las personas estrés, frustración y probablemente desencadene síntomas depresivos, lo que significa una patología con consecuencias catastróficas tanto para el trabajador como para la organización.,

Teniendo en cuenta que los riesgos psicosociales en el trabajo son actualmente una de las principales causas de enfermedades y de accidentes laborales, la conceptualización del síndrome Boreout invita a reflexionar sobre la monotonía, las condiciones en que 
aparece en un empleado, y, específicamente para la Psicología Laboral y Recursos Humanos, sobre la gestión del capital humano en las organizaciones, el liderazgo en las empresas, la relevancia de una acertada descripción de cargos y una correcta selección de personal (Latella y Velasco 2016).

\subsection{Síndrome de Boreout}

\subsubsection{Definición del concepto.}

No obstante, la relevancia que tiene el síndrome y los efectos que representa para la correcta gestión y desarrollo del capital humano en las organizaciones, se evidencia una muy escasa documentación científica sobre este fenómeno laboral que los últimos años afecta a una gran cantidad de empleados y conlleva como consecuencia la depresión y la falta de autoestima. Philippe Rothlin y Peter Werder (2011) son los primeros en describir este síndrome utilizando el término Boreout para identificarlo a partir de 2007.

De acuerdo con Pedro Gil-Monte, (2014), son evidentes los efectos negativos para una empresa el que sus trabajadores se encuentren en una situación de monotonía crónica en sus empleos; el capital humano se vería desperdiciado, los empleados se transformarían en talentos desperdiciados y la organización perdería efectividad.

\subsection{2 ¿Cómo se manifiesta este síndrome?}

Este aburrimiento crónico laboral se caracteriza por la escasez de desafíos, incentivos y de interés por parte del trabajador que lo padece; situación que implica un ámbito laboral en donde el empleado se siente excluido, se le exige mínimamente, está desmotivado y no tiene interés por realizar sus tareas. De acuerdo con Raquel Saralegui en su artículo Boreout, el síndrome del aburrimiento: "Paradójicamente aparentan estar muy ocupados para no asumir más trabajo y así refuerzan el aburrimiento". (Saralegui, 2010). Aparentemente es pereza u holgazanería, pero se tata del síndrome de Boreout, un nuevo nombre para un viejo mal laboral muy común en las oficinas, con perjuicio para la organización y para el trabajador.

El Boreout se compone de tres elementos que a continuación se explican de acuerdo con Philippe Rothlin y Peter Werder: a) Infraexigencia: Este concepto se relaciona con la sensación de poder rendir más de lo que la organización le exige o de lo que hace en su empleo.

b) Desinterés: Se percibe una falta de identificación con el puesto de trabajo.

c) Aburrimiento: Manifiesta un estado anímico de desgano, apatía y duda porque el empleado no sabe que hacer durante su horario laboral.

\subsection{Salud Ocupacional y el Boreout}

El estudio de la salud ocupacional en América Latina se remonta a las primas décadas del Siglo XX, cuando las empresas prestan mayor atención al compromiso de proteger a los trabajadores. La Salud Ocupacional como área de estudio cuenta con diferentes acepciones, aunque una de las más acertadas es la que propone la Organización Mundial de la Salud [OMS], que la conceptualiza como la "actividad multidisciplinaria dirigida a promover y proteger la salud de los trabajadores mediante la prevención, el control de enfermedades, accidentes, la eliminación de los factores y condiciones que ponen en peligro la salud y la seguridad en el trabajo" (OMS, 1995).

Es evidente que la vida cotidiana del ser humano presenta múltiples factores de estrés, como son un mal ambiente en casa, ruptura de pareja, etcétera. No obstante, Aranda, Pando, Torres, Salazar y Franco (2005) señalan que es en el entorno laboral donde se experimenta un acelerado incremento de estrés negativo (distrés) a causa de la deficiente estructura organizacional en el trabajo; por esta razón, se presenta el estrés en el trabajo como un problema en ascenso, con un costo personal, social y económico evidente (Sauter, Hurrell, Murphy y Levi, 1998). Por la gran cantidad de trabajadores afectados, se considera al estrés una de las problemáticas más significativas de salud en la actualidad. De acuerdo con la Agencia de información de la Unión Europea para la seguridad y la salud en el trabajo (EU-OSHA, 2019) los resultados de la Encuesta Europea de Empresas sobre Riesgos Nuevos y Emergentes 
(ESENER), arroja que el $79 \%$ de los empleados en Europa se encuentran afectados por estrés en sus espacios laborales.

Hasta hace pocos años uno de los principales síndromes en el trabajo era el Burnout: estado de agotamiento mental, emocional y físico que se presenta como resultado de exigir demasiado a los empleados, El síndrome Boreout vino a sumarse al Burnout; y en ese sentido, es importante diferenciar ambos padecimientos. En el boreout existe una falta de identificación en relación al empleo y una baja motivación; en tanto que en el síndrome del burnout el individuo se siente comúnmente quemada o totalmente exhausta. Un persistente boreout puede desencadenar la manifestación de estrés crónico que actúe como factor de riesgo para desarrollar burnout (Beltrán, Contreras, Forero, \& García, 2016). Por consiguiente, un boreout puede llegar a convertirse en un burnout. Entonces... ¿qué causa el boreout?

\subsubsection{Las causas del Boreout}

No hacer nada en el trabajo pudiera ser el sueño de muchos empleados, pero resulta que es realmente utópico pues la realidad es completamente diferente: el estar en el cargo laboral durante horas sin saber qué hacer es una situación desesperante (Cabrera, 2014). Es importante tener presente que el aburrimiento crónico en el ámbito laboral está relacionado con la percepción que tiene el trabajador de querer elevar su desempeño, poner a prueba sus capacidades, potencializarse profesionalmente, demostrar su crecimiento y no poder hacerlo resulta frustrante para el trabajador.

Según Rothlin y Werder (2011), este síndrome se produce por varias causas:

- Realización de tareas demasiado monótonas y aburridas, las personas que padecen el síndrome de boreout se sienten, generalmente, infrautilizadas.

- Empleo con actividades muy repetitivas.

- El empleado se encuentra en un trabajo donde no se siente motivado o donde sus expectativas no concuerdan con el puesto para el que fue contratado.

- Trabajar únicamente para pagar deudas.

- Ambigüedad en el rol por falta de precisión al determinar las funciones propias del puesto de trabajo. El empleado no tienes en claro cuál es su función en la empresa.

- Realización de funciones por debajo de las competencias del trabajador. Necesita mayores responsabilidades que se adapten a sus capacidades.

- La estructura jerárquica o el estilo de liderar de los gerentes que impide a los colaboradores intervenir en la toma de decisiones o desarrollar su potencial dentro de la organización.

- Inviabilidad para conseguir ascender en su posición dentro de la organización o de incrementar su salario.

- No visualizar un futuro en la empresa.

- No recibir algún ascenso prometido.

- Desmotivación o necesidad de reconocimiento a su trabajo por parte de sus superiores, por lo que el empleado considera inequitativo su esfuerzo en el trabajo.

- El trabajador no se siente preparado para cubrir el puesto.

Según el Médico en Salud Mental y Psicopatología Clínica, Dr. Guillermo Laich, (2012), las constantes exigencias a las que se ven sometidas las empresas traen por consecuencia nuevas trasformaciones en los modos y configuraciones del trabajo. De esta forma, han surgido desajustes entre el desempeño de los empleados y los requerimientos que los puestos de trabajo demandan. Tales discrepancias provocan la aparición de fenómenos negativos para los individuos ya que inciden en su bienestar, satisfacción, desempeño y calidad de vida laboral, en consecuencia, la organización se ve perjudicada en los niveles de productividad. El trabajador suele ser etiquetado como flojo y de esta forma se le asigna completamente la responsabilidad, deslindando a la organización de culpa alguna en la provocación de ese fenómeno (Aranda et al, 2005).

\subsubsection{La prevención del Boreout}

Es muy común el síndrome boreout en empleos con infraexigencia o donde solo se requiera la presencia del colaborador. Esto suele suceder en trabajos de vigilancia o de seguridad, donde solo se requiere la actuación 
del empleado en situaciones poco comunes en las que ocurra un imprevisto, y cuyo encargo consiste más en la observación o inspección permanente que en el propio desarrollo de habilidades determinadas. No obstante, para aquellos empleos que necesitan de una capacitación y, sobremodo, un espíritu innovador, la monotonía suele ser un gran motivo de frustración para el profesionista, aún más que la sobre-exigencia (Rothlin, y Werder, 2011).

Es relevante considerar que el boreout suele manifestarse casi en cualquier clase de trabajador, por lo menos una vez en su vida laboral. Profesionistas como periodistas, arquitectos, ingenieros, y maestros pueden verse afectados con mayor factibilidad si sus empleos no les brindan la oportunidad de desarrollar todo su potencial. De la misma manera, pueden desplegar este síndrome los vendedores, cajeros, oficinistas y aquellos que efectúen tareas repetitivas, o donde al trabajador no se le fomente la iniciativa y la creatividad. De tal relevancia es este fenómeno que, según estudios realizados, el $15 \%$ del personal de las oficinas está en camino de padecer este mal (Salary.com, 2005).

Ahora bien, las medidas para prevenir el síndrome boreout deben estar a cargo tanto por la empresa como por propio trabajador. Las acciones que puede adoptar la organización, de acuerdo con Guillermo Laich, (2012), tienen que ver con llevar a cabo un buen proceso de gestión de capital humano:

a) Diseño del puesto de trabajo. -

Es importante que se identifiquen claramente las habilidades y actitudes requeridas para ejercer las funciones en el cargo: cuáles son los objetivos propuestos y metas del puesto. Esto le permitirá a la empresa no tener equivocaciones en la contratación del personal. Asimismo, evitará generar expectativas erróneas en el contratado, obligándolo a realizar un trabajo para el cual no está preparado (Robbins y Coulter, 2018).

b) Evaluación de puestos. -

Atribución consistente en valorar cada cargo, de acuerdo con su relevancia y contribución a los intereses de una empresa. Para esto es necesario que se señalen los perfiles profesionales óptimos con los que se supondría se tenga un buen desempeño en él, como, por ejemplo: responsabilidades, habilidades requeridas, tipo de esfuerzo, etc. (Robbins y Coulter, 2018).

c) Selección de personal. -

El propósito del procedimiento consiste en seleccionar a los postulantes que demuestren las cualidades y experiencia profesional más apropiadas para el desempeño del puesto solicitado por la compañía (Arciniega, 2012). Contar con un método adecuado de selección de personal es trascendental para una organización, pues debe tenerse presente que:

- No existe el trabajador bueno o malo, sino el empleado correcto para el cargo adecuado en la empresa.

- Los trabajadores serán exitosos en su trabajo si cuentan con las habilidades y la motivación suficiente para cumplir todas sus funciones.

- Examinar y valorar las capacidades innatas del candidato, así como su motivación es fundamental para asegurar que el postulante al empleo es el adecuado y estará en la posición correcta.

d) Retención del capital humano

Es importante capacitar a la alta gerencia para que sean mejores coachs, de tal manera que colaboren y guíen a sus trabajadores a descubrir y desarrollar todo su potencial. Asimismo, la organización tiene que llevar a cabo planes de motivación y mejorar la comunicación interna, así como el ambiente laboral promoviendo el trabajo en equipo para que con esto sus colaboradores se puedan sentir cómodos en sus puestos de trabajo (Robbins y Coulter, 2018).

\section{MÉTODO}

\subsection{Problema.}

El estrés y la satisfacción laboral constituyen uno de los principales problemas y desafíos del área de gestión de personal, es por esto por lo que constituye un factor relevante en los últimos tiempos en el contexto laboral.

El Boreout constituye un novedoso riesgo psicosocial al que enfrentan muchos trabajadores. Se empieza a emplear este término en marzo del 2007 por los suizos Philippe Rothlin y Peter R. Werder en su libro publicado en esa fecha, en el cual se detalla y 
describe la patología laboral de quien se aburre en el trabajo. Es una situación totalmente nociva que afecta tanto al individuo que lo sufre, como a la organización para la cual labora.

El boreout, o síndrome de aburrimiento, se caracteriza por la falta de desafíos profesionales y de interés en el trabajo. Considerando que las nuevas generaciones se caracterizan por la búsqueda constante de nuevos desafíos y proyectos, el boreout se presenta actualmente como una amenaza concreta para el clima laboral y la moral del personal.

El boreout es mucho más frecuente de lo que parece y posee múltiples aristas. Para entender mejor este fenómeno, surge la siguiente interrogante: ¿Cómo incide el Síndrome Boreout en la gestión de capital humano?

\subsection{Hipótesis.}

Son las organizaciones las que propician e impulsan la aparición del síndrome Boreout ante una falta de dirección del talento del capital humano.

\subsection{Objetivos del Estudio.}

- Analizar el estado del desarrollo conceptual, los enfoques teóricos, tendencias y perspectivas acerca del síndrome laboral Boreout.

- Conocer la incidencia de la gestión de capital humano en la prevención del Síndrome Boreout.

\subsection{Justificación. -}

En la actualidad, la mayoría de las empresas coinciden en que solo aquellas que seleccionan adecuadamente al recurso humano y lo colocan correctamente dentro de su estrategia de negocio, contarán con la fortaleza para ser una organización efectiva y competitiva.

Cada vez se les otorga mayor relevancia a las acciones para maximizar la eficacia y eficiencia del empleado, así como para mantenerlo motivado y productivo, para poder aprovechar al máximo este activo de la empresa, el cual tiene una incidencia directa y clave en la competitividad y rentabilidad de la organización a la que pertenece.

La relevancia del estudio radica en explicar la correlación existente entre las gestiones de capital humano y la prevención del Síndrome Boreout.

\subsection{Diseño de Investigación.}

La metodología utilizada en esta investigación por sus características corresponde a un enfoque del tipo cualitativa y descriptiva con un alcance exploratorio, considerando que la misma está orientada a describir las causas del síndrome Boreout, así como sus consecuencias para los empleados y la gestión de capital humano en la prevención de este.

Es una investigación de tipo documental cuyo objetivo es recobrar de manera sistemática y reflexiva, el conocimiento que se ha acrecentado sobre el objeto de estudio, Síndrome Boreout, que por ser tan reciente aún no se ha investigado en profundidad.

\section{RESULTADOS}

Después de un exhausto análisis de la literatura, en inglés y español, en lo relativo al Síndrome de Boreout, se puede inferir que el aburrimiento crónico en el trabajo va más allá de la sensación de tedio o fatiga, que todos los trabajadores han sentido alguna vez; tampoco tiene que ver con estar desinteresados en las tareas laborales, sino que sobrepasa la experiencia que siente el empleado de haberse desconectado del trabajo para el que fue seleccionado, pues este ya no satisface su objetivo de realización personal y profesional, y ya no hay prevalencia entre el cargo y el proyecto de vida, en un sentido motivacional.

El categorizar como un síndrome el fenómeno de aburrimiento crónico en el trabajo, resulta relevante para que los gerentes del departamento de recursos humanos en las organizaciones analicen los factores facilitadores de las condiciones para el síndrome y para que consideren medidas de prevención en los contextos laborales.

No obstante, lo anterior, el fenómeno tipificado por sobrecarga laboral (síndrome burnout); atrae mayor atención que aquellos 
provocados por escaza exigencia al trabajador. Al realizar la búsqueda y estudio de materiales bibliográficos del tema síndrome laboral Boreout, se detecta que todos los autores retoman la conceptualización de Rothlin y Werder (2011), y actualmente aún resulta muy escaza la investigación sobre este fenómeno y su conceptualización. Si bien se han realizado algunas producciones relacionadas con el diseño y validación de instrumentos para la medición del síndrome Boreout, como las de Cabrera (2014), Azabache (2016), Beltrán et al. (2016); todavía no se han hecho valoraciones que sean significativas para establecer condiciones determinantes en el surgimiento y desarrollo del síndrome. En este aspecto, aunque se ha logrado detectar diversas condiciones laborales que facilitan el surgimiento del síndrome, no se encontró algún estudio de que hayan sido vinculadas o valoradas en relación directa con las características de personalidad de los trabajadores.

Sería pertinente un análisis de la prevalencia directa entre la tendencia al aburrimiento de algunas personas y la propensión de que desarrollen Boreout, de acuerdo con ciertas condiciones laborales. Además, la investigación documental no encontró propuestas que determinen de manera precisa las estrategias para evitar o prevenir el síndrome, tampoco la forma en que estas estarían interrelacionadas, y podrían aproximadamente efectivas, de acuerdo a determinadas estructuras organizacionales.

Aranda et al (2017), analizan cómo las organizaciones con estructuras rígidas donde no prestan atención a la gestión de capital humano pueden constituir un gran riesgo para la aparición de las condiciones del surgimiento de este tipo de fenómeno. En tal planteamiento, se pueden considerar las propuestas de diferentes autores, como Rothlin y Werder (2011), Cabrera (2014) y Beltrán et al. (2016), en las que los factores de prevención planteados se parecen enormemente a las características de una correcta administración de recursos humanos, sin embargo, en ninguno se infiere su relación con la aparición del síndrome Boreout, a partir de investigaciones experimentales.

Aun cuando en la literatura analizada no se menciona de manera explícita, el contrato psicológico podría ser un factor significativo a considerar en la manifestación del Boreout, toda vez que si existiera un incumplimiento del mismo por parte de la empresa, y esto provoca que se reduzca el carácter de proyección a futuro ofrecido para el puesto, el empleado puede empezar a evaluar negativamente el aporte de su empleador a sus proyectos y propósitos personales, y traer por consecuencia una rotura en la identificación de sí mismo con la compañía.

La baja de exigencia, señalada como un factor determinante del Boreout, y vinculada con la información recabada por Rothlin y Werder, en la que los empleados encuestados utilizaban aproximadamente dos horas de su jornada laboral en actividades personales, reorienta el focus a lo relativo a la gestión del talento del capital humano, asimismo a lo concerniente a la distribución de tareas, y conlleva a la propuesta de un planteamiento en la gestión del recurso humano como medida de prevención al Boreout.

Para futuras investigaciones empíricas se abre la posibilidad de analizar e indagar los factores psicosociales y organizacionales que pueden disminuir la sensación de aburrimiento en el ámbito laboral.

\section{CONCLUSIONES}

Una errónea descripción de puestos, así como un equivocado proceso de selección de personal puede tener consecuencias catastróficas, tanto para el empleado como para la organización; por un lado, un trabajador aburrido en las actividades laborales para las que fue contratado, porque el empleo no resultó ser equiparable a lo que estaba planteado en la descripción de puestos, y, por otro lado, un empleado que no llega a cumplir con las expectativas del puesto por no ser el candidato idóneo y no estar lo suficiente calificado para esa tarea. 
Un mal desempeño en el proceso de selección de personal puede afectar negativamente en la organización, además de repercutir en una pérdida económica debido al costo de rotación de un trabajador.

De la misma manera ocurre, como paso anterior a la selección, cuando se debe definir un perfil de puesto. Generalmente el problema se centra en definir el puesto y luego describir el perfil. Ambos conceptos no deben confundirse. La descripción del puesto consiste en señalar el nombre del cargo y las funciones o actividades a desempeñar, mientras que el perfil del candidato comprende los datos personales, su educación y su experiencia.

En concordancia al análisis llevado a cabo y las inferencias, se indican las siguientes recomendaciones en base a la teoría de comportamiento en las organizaciones planteada por Robbins (2009):

- Incluir la misión, las metas, la política y los propósitos fundamentados en la prevención de riesgos psicosociales, los cuales sean instituidos en todos los niveles de la empresa. Con lo anterior, se declara el compromiso de la empresa por mantener la salud mental de sus empleados.

- Difundir campañas que tengan como propósito informar a los empleados en qué consiste el Síndrome de Boreout; y plantear estrategias para que los trabajadores puedan prevenir y tratar adecuadamente el síndrome en caso de que se presente.

- Gestionar las funciones y puestos en relación a las habilidades técnicas, cognitivas e interpersonales que posean los trabajadores, de esta forma se seleccionará a la persona apropiada para cada puesto, y con ello, se podrán minimizar los niveles de estrés, disminuyendo la probabilidad de manifestarse el Boreout, ya que el individuo tendrá las capacidades para hacer frente los desafíos de su empleo.

- Implementar horarios más flexibles basándose en la planificación por objetivos. De esta manera los miembros de una empresa pueden identificar los objetivos y planear estrategias conforme en sus responsabilidades y en términos de los resultados esperados. Conforme a lo anterior, se podrán alcanzar los objetivos eficazmente, se reducirán los "tiempos muertos" (principal causa del Boreout), se aumentará el desempeño y se disminuirá el ausentismo laboral.

- Incluir Cursos Inductivos que incorporen dentro de sus temas una sección para riesgos psicosociales. De tal manera que los nuevos trabajadores al momento de ingresar a la empresa conozcan sobre el riesgo que implican los síndromes psicosociales en el ámbito laboral.

- Instaurar vínculos de comunicación directa en la organización. De esta manera se impedirán los obstáculos propios de la estructura jerárquica, permitiendo una mayor fluidez de comunicación entre los trabajadores y la alta gerencia.

- Establecer prácticas de relajación y técnicas de control de la respiración antes y después de la jornada laboral. De esta manera se ayudará a los empleados a controlar sus niveles de ansiedad y estrés, y los empleados podrán concentrarse en sus funciones y obligaciones; además, posibilitará la relajación después de su jornada laboral.

- Capacitar a los empleados en el uso efectivo de pausas activas en el trabajo. De tal manera que los colaboradores estén informados de cuando es necesario realizar una pausa a su trabajo, permitiendo la reducción de la tensión, elevando los niveles de concentración y evitando el tedio en el trabajo.

- Examinar la salud mental de los colaboradores de manera periódica, a través de charlas con el psicólogo de la compañía, o bien, con el trabajador social o directamente con el departamento de recursos humanos. Por medio de las evaluaciones se controlará la salud mental, previendo riesgos psicosociales. 


\section{REFERENCIAS}

Aranda, C., Pando, M., Torres, T. Salazar, J. y Franco, S. (2005). Factores Psicosociales laborales y síndrome de Burnout en médicos de familia. Revista de investigación. Universidad Nacional Mayor de San Marcos. 225-231. Recuperado de: https://www.redalyc.org/pdf/379/37966306.pdf

Arciniega, R. (2012). Factores organizacionales causantes del estrés en el trabajo y estrategias para afrontarlo. Revista Venezolana de Gerencia. 17(60): 611-634. Recuperado de: https://www.redalyc.org/pdf/290/29024892005.pdf

Azabache, K. (2016). Construcción y propiedades psicométricas de la Escala del Síndrome de Boreout en colaboradores adultos de Trujillo. Tesis para obtener el grado académico de doctor/a en psicología. Escuela de Postgrado. Universidad César Vallejo, Perú. Recuperado de: https://repositorio.ucv.edu.pe/handle/20.500.12692/186

Bahamondes, G. (2018). Factores de riesgo derivados de la Organización del trabajo en profesionales informáticos de la provincia del Biobío. Tesis para optar al Título de Ingeniero en Prevención de Riesgos. Escuela de Ciencias y Tecnologías. Departamento de Ciencias y Tecnología Vegetal. Universidad de Concepción Campus Los Ángeles. Chile. Recuperado de: http://repositorio.udec.cl/bitstream/11594/3318/4/Rivas\%20R\%C3\%ADos\%2C\%20Om ar.pdf

Beltrán, A. Contreras, B. Forero, L. y García, A. (2016). Game-mover: Herramienta gamificada para el diagnóstico del Síndrome de Boreout. Tesis de Grado. Facultad de Psicología. Universidad Católica de Colombia. Recuperado de: https://repository.ucatolica.edu.co/bitstream/10983/6097/4/Game-

Mover\%20\%20herramienta\%20gamificada\%20para\%20el\%20diagnostico\%20del\%20b oreout.pdf

Cabrera, L. (2014). Síndrome de Boreout: Diseño, confiabilidad y validación preliminar de un instrumento para su medición. Revista de la Universidad Industrial de Santander. $\begin{array}{lllll}\text { Colombia. } & 46 & \text { (3): 259-265. Recuperado de: }\end{array}$ https://www.redalyc.org/pdf/3438/343838645006.pdf

EU-OSHA (Agencia de información de la Unión Europea para la seguridad y la salud en el trabajo). (2019). Cómo gestionan los lugares de trabajo de Europa la seguridad y la salud. Recuperado de: https://visualisation.osha.europa.eu/esener\#!/es/survey/overview/2019

García, B., Villavicencio, E., García, M. (2019). Síndrome de Boreout en trabajadores baby boomers, x y millennials de la Ciudad de México. Psicología Iberoamericana, vol. 26, núm. 2, pp. 88-95, 2018. Recuperado de https://www.redalyc.org/jatsRepo/1339/133959841010/html/index.html\#: :text=Dentro \%20de\%20estos\%20casos\%20se,Rothlin\%20\%26\%20Werder\%2C\%202009).

Gil-Monte P. (2014). Psicosociología aplicada al estudio de los riesgos laborales: estrés y estrategias de afrontamiento. Manual de psicosociología aplicada al trabajo y a la prevención de los riesgos laborales (pp. 25-48). Madrid: Pirámide.

Laich, G. (2012). El ABC del Boreout. Recuperado de: http://www.guillermolaich.com/n/17/abcdel-boreout/lang/es

Latella, S., Marciano, N. y Velasco, E. (2016). Bore Out: Aburrimiento crónico en el trabajo. Trabajo de investigación final para optar al Título de Licenciado en Administración de Empresas. Unidad de Ciencias Económicas. Universidad Argentina de la Empresa. Recuperado de: https://repositorio.uade.edu.ar/xmlui/handle/123456789/5034

López, R. (2010). La selección de personal basada en competencias y su relación con la eficacia organizacional. Revista Perspectivas. Universidad Católica Boliviana San Pablo. Bolivia. 1(26): 129-152. Recuperado de: https://www.redalyc.org/pdf/4259/425941230007.pdf

Organización Mundial de la Salud (1995). Salud Ocupacional para Todos. Suiza Ginebra. Recuperado de: http://apps.who.int/iris/bitstream/10665/42109/1/951802071X_spa.pdf 
Peiró, J. (2008). Estrés laboral, liderazgo y salud organizacional. Revista: Papeles del Psicólogo. 68-82. Recuperado de: https://www.redalyc.org/pdf/778/77829109.pdf

Robbins, S. y Coulter, M. (2018). Administración 13E. Editorial: Pearson.

Rothlin, P. y Werder, P. (2011). El nuevo síndrome laboral: Boreout. Recupera la motivación. Editorial Penguin Ramdon House. Barcelona. España. p 16.

Saralegui R. Boreout, (2010). El síndrome del aburrimiento. Diario La Nación, nov 21; Sección Economía. En: http://www.lanacion.com.ar/1326923-boreout-el-sindrome-delaburrimiento

Salary.Com. (2005). El tiempo perdido en el trabajo cuesta miles de millones a las empresas, por Dan Malachowski. Recuperado de: http://www.salary.com/careers/layouthtmls/crel_display_nocat_Ser374_Par555.html

Sauter, S., Hurrell, J., Murphy, L., y Levi, L. (1998). Factores psicosociales y de organización. En O. I. Trabajo, Enciclopedia de salud y seguridad en el trabajo, (pp. 2-34). Ginebra: Organización Internacional del Trabajo. 